\title{
Neutrino Probes of Galactic and Extragalactic Supernovae
}

\author{
Shin'ichiro Ando \\ California Institute of Technology, Mail Code 130-33, Pasadena, CA 91125
}

\begin{abstract}
Neutrinos are a messenger of extreme condition inside a supernova core and a new-born neutron star. Since current ground-based detectors have potential to detect $\sim 10,000$ neutrinos from supernova at the galactic center, they could tell us lots of important physics. It includes: explosion mechanism, shock wave propagation, core temperature, and gravitational binding energy, as well as neutrino properties as elementary particle. In addition to the galactic supernova neutrino burst, one can still learn about them with diffuse supernova neutrino background, which is also soon to be detected. We review current situation from both points of view, and discuss prospects for future neutrino astrophysics.
\end{abstract}

Keywords: supernovae; neutrinos; diffuse background; elementary particle physics PACS: $97.60 . \mathrm{Bw} ; 98.70 . \mathrm{Vc} ; 13.15 .+\mathrm{g} ; 14.60 . \mathrm{Pq} ; 95.85 . \mathrm{Ry}$

\section{INTRODUCTION}

A core-collapse supernova explosion is one of the most spectacular events in astrophysics, and it attracts a great deal of attention from many physicists and astronomers. It also produces a number of neutrinos; $99 \%$ of its gravitational binding energy is transformed to neutrinos. Therefore, neutrinos play an essential role in supernovae. For example, neutrino interaction might be affecting the core-collapse dynamics, by causing the observed kick velocity of newly born neutron stars (see, e.g., Ando [1], and references therein).

However, how core-collapse supernovae explode still remains one of the greatest mysteries in astrophysics. Nuclear fusion reactions in the core of a massive star produce progressively heavier elements until a Chandrasekhar mass of iron is formed, and electron degeneracy pressure cannot support the core under the weight of the stellar envelope. The core collapses until it reaches nuclear densities and neutrino emission begins; then an outgoing bounce shock should form, unbinding the envelope and producing the optical supernova. While this is successful in nature, in most numerical supernova models, the shock stalls, so that the fate of the entire star is to produce a black hole (after substantial neutrino emission), but no optical supernova.

Since the gravitational energy release transferred to neutrinos, about $3 \times 10^{53} \mathrm{erg}$, is $\sim 100$ times greater than the required kinetic energy for the explosion, it is thought that neutrino emission and interactions are a key diagnostic or ingredient of success. However, very little is directly known about the total energies and temperatures of the neutrino flavors. The $\simeq 20$ events from SN 1987A were only crudely consistent with expectations for $\overline{\mathrm{v}}_{e}$, and gave very little information on the other flavors. It is thus essential to collect more supernova neutrino events to understand the supernova 
physics more precisely. More concretely, we are eager to know what the explosion mechanism is, what the initial conditions of new-born protoneutron stars would be. Neutrinos potentially give us the answers (or at least implications) for these questions, since they are the direct messenger of the collapsing cores unlike photons. Furthermore, we can learn neutrino properties with supernova neutrinos, and this would be fascinating for the particle physics community. With the progressive results by many experiments, we learned a lot about neutrino physics and most of them were quite surprising, but still, only very little is known about the neutrino sector. Supernova neutrinos can play a unique role for us to learn more about neutrinos because of the extreme and (therefore) unique conditions of the core, from which neutrinos are produced.

At present, several large-volume underground detectors are running. SuperKamiokande (SK), which is at the Kamioka mine in Japan, is the representative Čerenkov detector, filled with 50-kton pure water (32-kton fiducial volume for the burst mode and 22.5-kton for other modes). In addition to SK, there are many detectors of various types; e.g., Sudbury Neutrino Observatory (SNO; heavy water Čerenkov detector) and KamLAND (scintillator) both having $\sim 1$-kton fiducial volume. With these detectors, we have made remarkable progress on the neutrino physics especially about flavor mixing, or neutrino oscillation, using the atmospheric, solar, and reactor neutrino data. Furthermore, as a fascinating target of these detectors, a supernova neutrino burst has been considered by many researchers for many years. If a core-collapse supernova explosion occurs in the galaxy (e.g., at $10 \mathrm{kpc}$ from the Earth), SK is expected to detect about 10,000 neutrinos (mainly $\overline{\mathrm{v}}_{e}$ events), and SNO and KamLAND would also detect several hundreds of events (mainly $v_{e}$ and $\bar{v}_{e}$ for SNO, and $\bar{v}_{e}$ for KamLAND). Because the extreme physical conditions realize inside the supernova core such as very high density and magnetic fields, and neutrinos escape directly from the core unlike photons, then they would be a unique probe of these environments. We note that the order of 10,000 events would be enough for statistically significant analysis.

On the other hand, it is not strange at all even if there occurs no supernovae in our galaxy in the near future (while detectors are running). This is because the supernova rate is estimated to be a few per century. Even under such discouraging situation, we can still learn physics through supernova neutrinos, using diffuse neutrino background from cosmological supernovae. In terms of statistics, this is not competitive to the galactic burst; nevertheless, it is important because it could be the only method to probe supernova core directly with neutrinos. Furthermore, this could tell us what the average neutrino emission spectrum would be, which would turn out to be the average condition of supernova core. This could also provide unique method for particle astrophysics and cosmology including test of long range neutrino lifetime and probe of cosmic star formation history.

The aim of this contribution is to reveal the fundamental physics with supernova neutrinos, ranging from supernova physics such as explosion mechanisms to neutrino properties as the elementary particle. More precisely, we focus on future prospects. This is partly because we still do not have any data of supernova neutrinos except for $\sim 20$ events from SN 1987A detected by Kamiokande-II [2] and IMB [3]; the analysis of these $\sim 20$ data has been performed and several implications for supernova and neutrino physics have been discussed by many researchers. To what extent we can learn from the future supernova neutrino observation is the main thrust, and we visit this point 
by studying two different categories - galactic and cosmological supernovae-in the following discussions.

\section{IMPLICATIONS FROM THE GALACTIC SUPERNOVA NEUTRINO BURST}

In this section, we discuss several possible implications from the future galactic supernova neutrino burst as well as the existing neutrino data from SN 1987A. Using the currently working detectors such as SK, we can obtain the distributions of energy, time, and direction of incident neutrinos, from each of which we can derive useful information as detailed below.

\subsection{Supernova neutrino spectrum}

\subsubsection{Temperature and binding energy of protoneutron stars}

It is an intriguing question what the temperature and gravitational binding energy of the newly born protoneutron stars are. That information then implies the mass and radius of the star, telling us the equation of state of very high-dense nuclear matter. Since the expected number of events are $\sim 10,000$, we can precisely construct the incident neutrino energy distribution, which reflects the physical condition of the protoneutron stars, such as temperature. Furthermore, by analyzing time evolution of the detected spectrum, we can follow the evolution of the temperatures and radius of the protoneutron star. It is also worth mentioning the result of supernova neutrino burst from SN 1987A obtained by Kamiokande-II and IMB. Many researchers analyzed these data, and obtained some implications for the supernova parameters (see, e.g., Refs. [4, 5, 6], and references therein).

\subsubsection{Neutrino mixing parameters}

Neutrinos undergo flavor mixing while they propagate the stellar envelope, in some characteristic ways. As the result, the neutrino spectrum (mainly $\bar{v}_{e}$ in the case of $\mathrm{SK})$ at the Earth is quite different from the original one. Since we know some mixing parameters very precisely, we can reconstruct the original spectrum from the obtained one to obtain the information on physical parameters of the supernova.

Another possibility is to derive the information on unconstrained mixing parameters such as $\theta_{13}$ and mass hierarchy from the obtained neutrino spectrum [7, 8, 9]. Especially if the mass hierarchy is inverted, the complete conversion between $\bar{v}_{e}$ and $\bar{v}_{\mu, \tau}$ could occur, enhancing the high-energy tail of the spectrum predicting large number of events. In the case of normal mass hierarchy, on the other hand, SK has little sensitivity to the value of $\theta_{13}$ since it mainly captures $\bar{v}_{e}$. In this case, SNO plays an important role, by efficiently collecting $v_{e}$ events, whose mixing scheme strongly relies on $\theta_{13}$. Therefore, 
a combined analysis of the SK and SNO (and other) data would become essential in evaluating the mixing parameters as well as the quantities regarding supernova physics. We can solve the degeneracy between models using both the $\mathrm{v}_{e}$ and $\bar{v}_{e}$ signals, although the statistics would not be great especially with SNO.

Since supernovae would be highly magnetized, we could also test the neutrino magnetic moment. This effect causes a similar effect as flavor oscillation, but it also changes spin, e.g., $\bar{v}_{e} \leftrightarrow v_{\mu}$ conversion. This spin-flavor conversion in the context of supernova neutrinos have been studied in Ref. $[10,11,12]$ (see also references therein).

\subsection{Time evolution of neutrino luminosity}

\subsubsection{Explosion mechanism}

From the neutrino luminosity curve, we study the dynamical evolution of the central remnant such as neutron stars. If the explosion proceeds by the delayed mechanism, we could identify the matter accretion phase, where neutrino luminosity becomes higher due to the shock stall and fall back of the matter. On the other hand, if the explosion is driven by the prompt mechanism without any shock stalls, then there should not be any luminosity enhancement; the protoneutron star cooling phase should start immediately [13]. We can also follow the evolution of the protoneutron stars by observing the latetime luminosity curve. In the case of the burst at $10 \mathrm{kpc}$, we would have enough statistics to follow the evolution precisely.

\subsubsection{Shock wave propagation}

Although neutrinos can be directly emitted from the core, we might be able to infer the shock wave propagation through the stellar envelope (at far outer region). This is because the neutrino mixing probability could change as the shock changes density profiles at the resonance region, and that signature might appear in the neutrino spectrum and luminosity curve (see, e.g., Ref. [14, 15, 16], and references therein). Furthermore, if the reverse shock forms when the supersonically expanding neutrino-driven wind collides with the slower earlier supernova ejecta that gives rise to a "double dip" feature in the number of events and average neutrino energy as a function of time [15]. These features are observable in the $\bar{v}_{e}$ signal for an inverted and in the $v_{e}$ signal for a normal neutrino mass hierarchy, provided $\sin ^{2} \theta_{13} \gg 10^{-5}$. This is because the higher resonance, which can be affected by the shock waves, would be relevant only in these cases.

\subsubsection{Black hole formation}

Black hole formation may be triggered by mass accretion or a sudden change in the high-density equation of state. If this transition occurs during the neutrino luminosity is still high, then the neutrino signal from the supernova will be terminated abruptly (the 
transition takes $<0.5 \mathrm{~ms}$ ). The properties and duration of the signal before the cutoff are important measures of both the physics and astrophysics of the cooling protoneutron star. Discussing more concretely, if the black hole formation occurs while the neutrino luminosity is still as high as $10^{52} \mathrm{erg} \mathrm{s}^{-1}$ (for the first $\sim 1 \mathrm{~s}$ ), the rate just before the cutoff (hereafter $t_{\mathrm{BH}}$ ) is about $1500 \mathrm{~s}^{-1}$ at $\mathrm{SK}$, in the case of supernova at $10 \mathrm{kpc}$. Even if it occurs at the late phase, when the luminosity is one order of magnitude smaller, the rate is $\simeq 150 \mathrm{~s}^{-1}$. After $t_{B H}$, the rates are zero. The $0.5 \mathrm{~ms}$ duration of the cutoff can be disregarded, which should contain about 0.4 events in the early case and about 0.04 events in the late case. Since these are fewer than 1, the cutoff can be considered to be sharp [17].

\subsection{Determination of the supernova direction}

Since neutrinos can immediately escape from the supernova core, contrary to the optical signal that is caused by the shock breakout from the photosphere, their detection tells us the upcoming optical explosion in advance by about a couple of hours. Therefore, it would be essential to ask how well the supernova can be located by its neutrino signal. Elastic scattering events ( $v e^{-} \rightarrow v e^{-}$) strongly peaks at the forward direction and should help locate the supernova. Using Monte Carlo simulations, we found that the supernova direction can be determined with the accuracy of about $10^{\circ}$ ([18]; see also, Refs. [19, 20]) in the case of supernova occurring at $10 \mathrm{kpc}$. This information would be informed to the astronomical community through the Supernova Early Warning System (SNEWS; [21]).

\section{DIFFUSE NEUTRINO BACKGROUND FROM COSMOLOGICAL SUPERNOVAE}

Because supernova explosions have occurred very commonly in both the past and present universe, tracing the cosmic star formation rate (SFR), they should have emitted a great number of neutrinos, which now make a diffuse background; we refer this as supernova relic neutrinos (SRNs). Involved physics in SRN ranges quite widelyfrom cosmic SFR and supernova physics to neutrino properties as elementary particles. Therefore, detecting SRNs or even setting limits on their flux can give us quite useful and unique implications for various fields of astrophysics, cosmology and particle physics. Detectability of SRNs in various detectors and its implications have been discussed in many theoretical papers from various points of view (see Ref. [22] for a review), about which we discuss in the following.

Flux estimation requires models of neutrino spectrum emitted from each supernova explosion and cosmic SFR. Furthermore, the effect of neutrino oscillation should now be taken into account appropriately. In addition to a flux estimation as precise as possible, a detailed discussion of background events, which hinder the SRN detection, is essential. A stringent observational upper limit on the SRN flux is obtained by the SK group [23] and its value is just above the recent theoretical predictions [24, 22]. In order to make the SRN detection more likely, a promising method was proposed by Beacom and Vagins [25]. Their basic idea is to dissolve $\mathrm{GdCl}_{3}$ into the water Čerenkov detectors, 
which greatly reduces the background events if it is applied to the currently working or proposed future detectors such as SK, Hyper-Kamiokande (HK) and Underground Nucleon Decay and Neutrino Observatory (UNO). Therefore, we are now at an exciting stage, where SRNs might soon be detected actually, and be used to obtain several implications for various fields of astrophysics as a unique and complementary method to usual observations of the light.

Before moving on to details, we briefly mention another possibility. In the intermediate regime between galactic and cosmological scales, megaton detectors could also detect supernova neutrinos from relatively nearby galaxies $(<10 \mathrm{Mpc})$ with a rate of $\sim 1 \mathrm{yr}^{-1}$ [26]. This could also help our understanding on supernova neutrinos together with galactic and cosmological signals.

\subsection{Flux and event rate of supernova relic neutrinos}

The cosmic SFR is being progressively established and is inferred from rest-frame UV, NIR H $\alpha$, and FIR/submillimeter observations. In the local universe, all studies show that the comoving SFR monotonically increases with $z$ out to a redshift of at least 1. The core-collapse supernova rate can be obtained either through the SFR, or by the direct number count of supernova. These different approaches give a consistent result, and therefore, we can trust them. Here we use the SFR model obtained by the GALEX satellite [27], and convert it to the supernova rate by assuming the Salpeter initial mass function.

As for the original supernova neutrino spectrum, here we use the result of numerical simulation by Ref. [13]. The effect of neutrino oscillation is also included, assuming the case of normal mass hierarchy.

Figure 1(a) shows the SRN flux as a function of neutrino energy. The flux of solar neutrinos, reactor, and atmospheric neutrinos, which becomes background events for SRN detection, is shown in the same figure. The SRN flux peaks around $5 \mathrm{MeV}$ and exponentially decreases above that energy. The energy range in which we are more interested is high-energy regions such as $E_{\mathrm{v}}>19.3 \mathrm{MeV}$ and $E_{\mathrm{v}}>11.3 \mathrm{MeV}$, because as discussed below, the background events are less critical and the reaction cross section increases as $\propto E_{v}^{2}$. In such a range, the SRN flux is found to be $5.5 \mathrm{~cm}^{-2} \mathrm{~s}^{-1}\left(E_{\mathrm{v}}>11.3\right.$ $\mathrm{MeV})$ and $1.1 \mathrm{~cm}^{-2} \mathrm{~s}^{-1}\left(E_{\mathrm{v}}>19.3 \mathrm{MeV}\right)$, for our model.

With the SK detector, whose fiducial volume is $22.5 \mathrm{kton}$, we might be able to detect SRNs. Furthermore, much larger water Čerenkov detectors such as HK and UNO are being planned. The expected event rates at such detectors are shown in Fig. 1(b) in units of $(22.5 \mathrm{kton} \mathrm{yr})^{-1} \mathrm{MeV}^{-1}$; with SK, it takes a year to obtain the shown SRN spectrum. The event rate integrated over various energy ranges is $5.5(22.5 \mathrm{kton} \mathrm{yr})^{-1}$ for $E_{e}>10 \mathrm{MeV}$ and $2.4(22.5 \mathrm{kton} \mathrm{yr})^{-1}$ for $E_{e}>18 \mathrm{MeV}$. This clearly indicates that if the background events that hinder the detection are negligible, the SK has already reached the required sensitivity for detecting SRNs. 

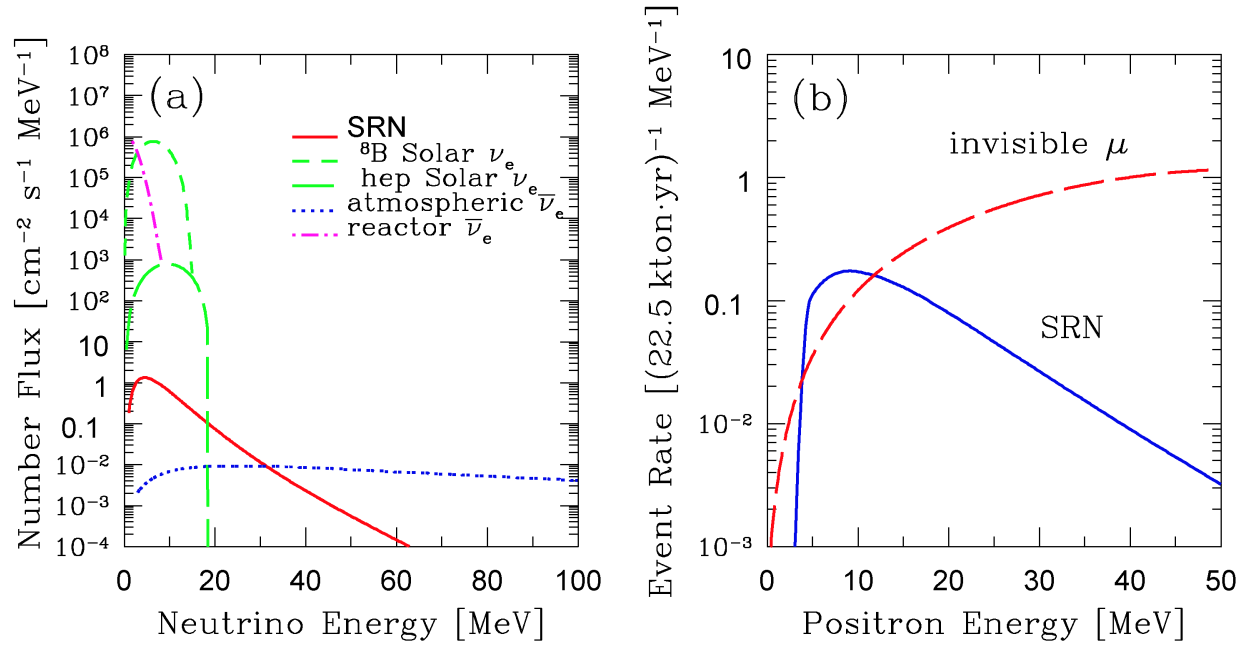

FIGURE 1. (a) Number flux of SRNs compared with other background neutrinos. (b) Event rate of SRNs and invisible muon decay products. From Ref. [22].

\subsection{Observational upper limit and implications}

The background events are atmospheric [28, 29] and solar neutrinos [30], antineutrinos from nuclear reactors [31], spallation products induced by cosmic-ray muons and decay products of invisible muons (e.g., [32]). We show in Figs. 1(a) and (b) the flux and event rate of these background events.

The most stringent upper limit on the SRN flux is obtained by the observation for 1496 days at the SK detector [23]. This limit is obtained by the statistical analysis including these background events from atmospheric neutrinos and invisible muons, and is $<1.2 \mathrm{~cm}^{-2} \mathrm{~s}^{-1}$ over the energy region of $E_{\mathrm{v}}>19.3 \mathrm{MeV}$ (90\% CL). Comparing with the prediction for the same energy range, we find that the current SK limit is just above the prediction. This strong constraint motivated many theoretical studies $[33,34,35,24,36,37,38,39,40]$ and has been translated into constraints on various quantities. The shaded region of Fig. 2(left) corresponds to the SK flux limit [23], reinterpreted in terms of the time-integrated luminosity $L_{v}$ and the spectrum average energy $\left\langle E_{v}\right\rangle$ for $\bar{v}_{e}$ [39]. Most numerical simulations predict around point $\mathrm{D}$ shown in the same figure, and hence, the current limit is encouragingly close to this "canonical" point.

In the near future, sensitivity of water Čerenkov detectors for the SRN detection would be significantly improved by the promising technique proposed recently [25]. The basic idea is the same as the delayed coincidence technique actually adopted at SNO or KamLAND, but $\mathrm{GdCl}_{3}$ is dissolved into the pure-water of SK (or other future detectors), which enables us to actively identify $\bar{v}_{e}$ by capturing neutrons produced by the $\overline{\mathrm{v}}_{e} p \rightarrow e^{+} n$ reaction. Owing to this proposal, the range $10-30 \mathrm{MeV}$ would 

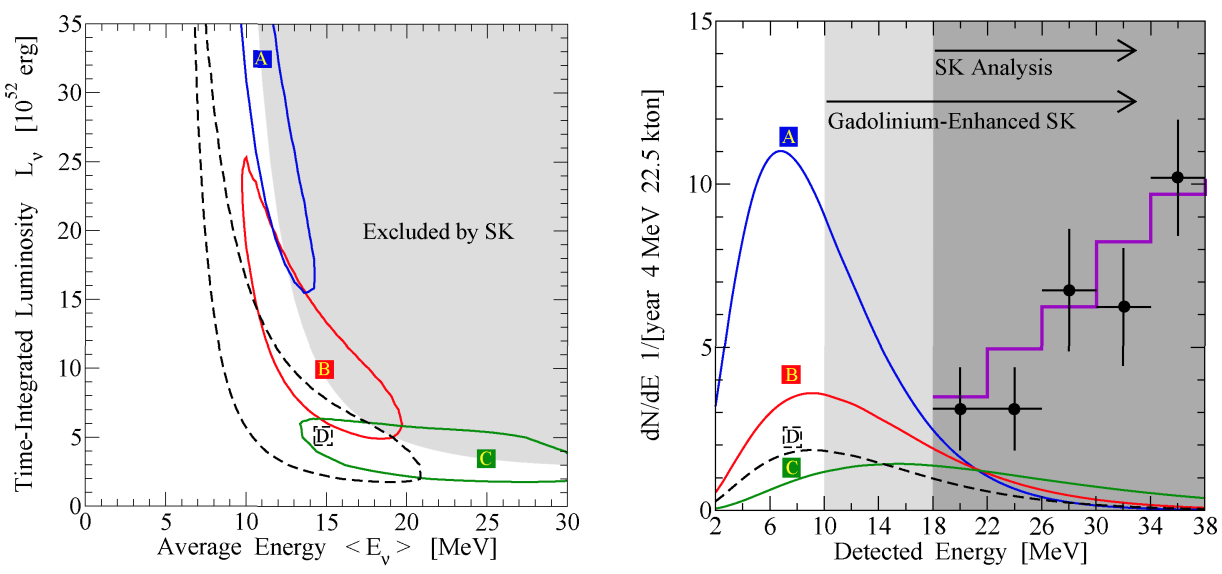

FIGURE 2. Left: Joint limits on the time-integrated luminosity $L_{v}$ and the spectrum average energy $\left\langle E_{\mathrm{v}}\right\rangle$ for $\overline{\mathrm{v}}_{e}$ due to the SRN non-detection (shaded region). Contours are possible $90 \%$ C.L. measurements of the emission parameters for each point A-D after 5 years running of a gadolinium-enhanced SK detector. Right: SRN detection spectra for points A-D (solid and dashed curves), efficiency-corrected SK data (points with error bars), and detector background (solid steps). From Ref. [39].

be an energy window because we can positively distinguish the $\bar{v}_{e}$ signal from other backgrounds such as solar neutrinos $\left(v_{e}\right)$, invisible muon events and spallation products. Figure 2(right) eloquently tells us how promising this proposal is for the SRN detection. SK is so far sensitive only to the dark shaded region of Fig. 2(right) above $18 \mathrm{MeV}$ due to high backgrounds at lower energies. With the addition of gadolinium, these backgrounds in the range $10-18 \mathrm{MeV}$ would be removed, and that shown would be reduced by a factor $\sim 5$, opening up the light shaded region for analysis. As the result, we can distinguish three typical models A-C quite well, as shown as contours in Fig. 2(left). We note that these models give almost the same yield at the current SK above $18 \mathrm{MeV}$. In addition, a model supported by many simulations (point $\mathrm{D}$ ) can also be probed quite well with five-year data.

\section{ACKNOWLEDGMENTS}

The author acknowledges support from Sherman Fairchild Foundation at Caltech.

\section{REFERENCES}

1. S. Ando, Phys. Rev. D 68, 063002 (2003).

2. K. Hirata, et al., Phys. Rev. Lett. 58, 1490 (1987).

3. R. M. Bionta, et al., Phys. Rev. Lett. 58, 1494 (1987).

4. B. Jegerlehner, F. Neubig, and G. Raffelt, Phys. Rev. D 54, 1194-1203 (1996).

5. M. Kachelriess, R. Tomas, and J. W. F. Valle, JHEP 01, 030 (2001).

6. A. Mirizzi, and G. G. Raffelt, Phys. Rev. D 72, 063001 (2005).

7. A. S. Dighe, and A. Y. Smirnov, Phys. Rev. D 62, 033007 (2000). 
8. K. Takahashi, and K. Sato, Prog. Theor. Phys. 109, 919 (2003).

9. C. Lunardini, and A. Y. Smirnov, J. Cosmol. Astropart. Phys. 06, 009 (2003).

10. S. Ando, and K. Sato, Phys. Rev. D 67, 023004 (2003).

11. S. Ando, and K. Sato, Phys. Rev. D 68, 023003 (2003).

12. S. Ando, and K. Sato, J. Cosmol. Astropart. Phys. 0310, 001 (2003).

13. T. Totani, K. Sato, H. E. Dalhed, and J. R. Wilson, Astrophys. J. 496, 216 (1998).

14. K. Takahashi, K. Sato, H. E. Dalhed, and J. R. Wilson, Astropart. Phys. 20, 189 (2003).

15. R. Tomàs, M. Kachelriess, G. G. Raffelt, A. Dighe, H.-T. Janka, and L. Scheck, J. Cosmol. Astropart. Phys. 0409, 015 (2004).

16. G. L. Fogli, E. Lisi, A. Mirizzi, and D. Montanino, J. Cosmol. Astropart. Phys. 0504, 002 (2005).

17. J. F. Beacom, R. N. Boyd, and A. Mezzacappa, Phys. Rev. D 63, 073011 (2001).

18. S. Ando, and K. Sato, Prog. Theor. Phys. 107, 957 (2002).

19. J. F. Beacom, and P. Vogel, Phys. Rev. D 60, 033007 (1999).

20. R. Tomàs, D. Semikoz, G. G. Raffelt, M. Kachelriess, and A. S. Dighe, Phys. Rev. D 68, 093013 (2003).

21. K. Scholberg (1999), astro-ph/9911359.

22. S. Ando, and K. Sato, New J. Phys. 6, 170 (2004).

23. M. Malek, et al., Phys. Rev. Lett. 90, 061101 (2003).

24. L. E. Strigari, M. Kaplinghat, G. Steigman, and T. P. Walker, J. Cosmol. Astropart. Phys. 0403, 007 (2004).

25. J. F. Beacom, and M. R. Vagins, Phys. Rev. Lett. 93, 171101 (2004).

26. S. Ando, J. F. Beacom, and H. Yuksel, Phys. Rev. Lett. 95, 171101 (2005).

27. D. Schiminovich, et al., Astrophys. J. 619, L47 (2005).

28. G. D. Barr, T. K. Gaisser, P. Lipari, S. Robbins, and T. Stanev, Phys. Rev. D 70, 023006 (2004).

29. M. Honda, T. Kajita, K. Kasahara, and S. Midorikawa, Phys. Rev. D 70, 043008 (2004).

30. J. N. Bahcall, Neutrino Astrophysics, Cambridge University Press, 1989.

31. C. Bemporad, G. Gratta, and P. Vogel, Rev. Mod. Phys. 74, 297 (2002).

32. S. Ando, K. Sato, and T. Totani, Astropart. Phys. 18, 307 (2003).

33. M. Fukugita, and M. Kawasaki, Mon. Not. R. Astron. Soc. 340, L7 (2003).

34. S. Ando, and K. Sato, Phys. Lett. B 559, 113 (2003).

35. S. Ando, Phys. Lett. B 570, 11 (2003).

36. S. Ando, Astrophys. J. 607, 20 (2004).

37. L. E. Strigari, J. F. Beacom, T. P. Walker, and P. Zhang, J. Cosmol. Astropart. Phys. 0504, 017 (2005).

38. J. F. Beacom, and L. E. Strigari, Phys. Rev. C 73, 035807 (2006).

39. H. Yüksel, S. Ando, and J. F. Beacom, Phys. Rev. C 74, 015803 (2006).

40. C. Lunardini, Phys. Rev. D 73, 083009 (2006). 